\title{
El cuarto advenimiento
}

\section{The fourth coming}

\author{
José Félix Patiño Restrepo*
}

https://doi.org/10.35454/rncm.v1n1.071

Hace cuarenta años, en marzo de 1978, J.C. Stevens envió una carta al Journal of the American Medical Association (JAMA) que tituló Surgical Nutrition, The fourth coming (1). (Nutrición Quirúrgica. El cuarto advenimiento, en mi traducción). "El advenimiento del soporte nutricional intensivo en pacientes desnutridos tanto en la fase preoperatoria como en la postoperatoria puede ser el «el cuarto advenimiento » como un avance mayor en el manejo de los pacientes quirúrgicos ocurrido en los últimos 150 años" (The advent of intensive nutritional support for nutritionally depleted preoperative and postoperative patients might well prove to be the "fourth coming" of a major advance in the care of surgical patients in the past 150 years).

Según el Diccionario de la Real Academia Española, el vocablo advenimiento tiene las siguientes acepciones:

1. Llegada de alguien o algo.

2. Acceso al cargo de un sumo pontífice o de un soberano al trono.

3. Suceso, acontecimiento.

Ciertamente el desarrollo de la nutrición parenteral representa la llegada de algo totalmente nuevo, un gran suceso, un acontecimiento en el devenir histórico de la medicina.

En su carta, Evans plantea que el Primer advenimiento fue el uso y desarrollo de la anestesia por Crawford W. Long en 1847 utilizando éter en pacientes obstétricas, incluyendo a su propia esposa. Hoy vemos como la cirugía bajo anestesia pasó de ser algo realmente bárbaro a un procedimiento indoloro y que permite al cirujano realizar la intervención con la amplitud necesaria.

El Segundo advenimiento ocurre en 1867 cuando Joseph Lister reconoce la importancia de la infección de la herida y propone los principios de la asepsia y la antisepsia.

El Tercer advenimiento es la aparición de los antibióticos, primero de la penicilina por Sir Alexander

\footnotetext{
* Jefe Honorario Departamento de Cirugía, Fundación Santa Fe de Bogotá, Bogotá, D.C, Colombia.
}

Fleming y asociados en 1928, lo cual significó la curación de infecciones y, especialmente, la prevención de la infección quirúrgica.

El Cuarto advenimiento, según Stevens, ocurre cuando en 1968 Stanley J. Dudrick y asociados proponen la nutrición parenteral total en pacientes con malfunción severa del tracto gastrointestinal o severamente catabólicos y demuestran acelerada cicatrización en casos que se caracterizaban por un mal pronóstico $^{(2)}$.

Tales advenimientos significan un progreso inmenso que se traduce en el salvamento de muchas vidas por la manera como se dispensa la atención médica, especialmente en el caso del paciente quirúrgico. El cuarto advenimiento, la nutrición parenteral, cambió el pronóstico de entidades nosológicas previamente asociadas a una elevada mortalidad, como lo eran, por ejemplo, las fístulas enterocutáneas. Y también dio lugar a la aparición de una nueva y bien estructurada profesión: la nutrición clínica.

Hoy, cincuenta años después de la publicación de Dudrick y colaboradores ${ }^{(2)}$, la nutrición parenteral es un formidable componente del armamentario terapéutico, especialmente en el cuidado del paciente en estado crítico.

El filósofo de la ciencia, físico e historiador norteamericano Thomas S. Kuhn (1922-1996) en su libro La Estructura de las Revoluciones Científicas (3) popularizó el término paradigma, que hoy se usa para significar la estructura de los supuestos básicos que definen o constituyen una teoría científica. Según el Diccionario de la Real Academia Española, el vocablo viene del latín tardío paradigma, y este del griego parádeigma. Estas son las tres primeras acepciones:

1. m. Ejemplo o ejemplar.

2. m. Teoría o conjunto de teorías cuyo núcleo central se acepta sin cuestionar y que suministra la base y modelo para resolver problemas y avanzar en el conocimiento. El paradigma Newtoniano. 
3. m. Ling. Relación de elementos que comparten un mismo contexto fonológico, morfológico o sintáctico en función de sus propiedades lingüísticas.

Según lo anterior, podemos denominar paradigma a cada uno de los advenimientos que describe Stevens, y resulta válido hablar del paradigma de la nutrición parenteral. Así lo han planteado Sanchez y Daly ${ }^{(4)}$.

Enorme ha sido el impacto del advenimiento dela nutrición parenteral (NP) desarrollada por Stanley J. Dudrick. En casos de pancreatitis aguda grave la NP logra preservar la composición corporal y un aumento significativo de los depósitos proteicos ${ }^{(5)}$; es la modalidad terapéutica primaria y esencial en casos de falla intestinal por fístulas enterocutáneas y otras causas, y hoy se administra con seguridad en el domicilio del paciente ${ }^{(6)}$; la Sociedad Europea de Nutrición Clínica y Metabolismo (ESPEN, por su nombre en inglés) ha publicado unas guías muy completas para el uso y la administración de la NP ${ }^{(7)}$.

Hoy la NP representa una formidable arma terapéutica, y combinada en la forma debida con la nutrición enteral, ha sido el factor salvador de miles de vidas.
El mismo S.J. Dudrick y J.M. Pimiento hacen reflexiones y revisan las controversias y desafíos de la Nutrición Parenteral ${ }^{(8)}$, ese gran cuarto advenimiento en la historia de la medicina.

\section{Referencias bibliográficas}

1 Stevens JC. Surgical Nutrition: The Fourth Coming. AMA. 1978; 239(3):192.

2 Dudrick SJ, Wilmore DW, Vars HM, et al. Long-term parenteral nutrition with growth, development and positive nitrogen balance. Surgery 1968;64: 134-42.

3 Kuhn TS. The Structure of Scientific Revolutions, 2nd Ed., Univ. of Chicago Press, Chicago \& London, 1970).

4. Sanchez JA, Daly JM, Dudrick SJ. A Paradigm Shift. Arch Surg. 2010; 145(6):512-4

5. Chandrasegaram MD, Plank LD, Windsor JA. The Impact of parenteral nutrition on the body composition of patients with acute pancreatitis. JPEN 2005;29(2):65-73.

6 Huisman-de Waal G, Schoonhoven L, Jansen J, Wanten G, Van Achterberg T. The impact of home parenteral nutrition on daily life-A review. Clin. Nutr. 2007;26(3):275-88.

7 Staun M, Pironi L, Bozzetti F, et al. ESPEN Guidelines on Parenteral Nutrition: Home Parenteral Nutrition (HPN) in adult patients. Clin. Nutr. 2009;28:467-79.

8 Dudrick SJ, Pimiento JM. Parenteral nutrition and nutritional support of surgical patients: reflections, controversies, and challenges. Surg Clin North Am. 2011;91(3):675-92.

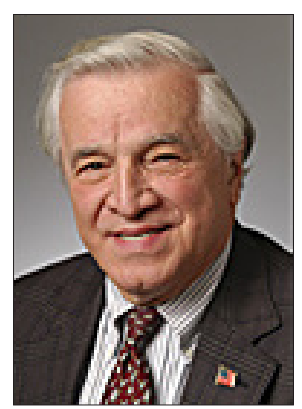

Stanley J Dudrick nació en Nanticoke, Pennsylvania el 9 de abril de 1935, obtuvo el grado de B.S. en Biología en el Marshall College y el título de médico en la Universidad de Pensilvania en 1961. Actualmente es Profesor Emérito de Cirugía en la Universidad de Yale. 